\title{
Multielectrode Analysis of Coordinated, Multisite, Rhythmic Bursting in Cultured CNS Monolayer Networks
}

\author{
Michael H. Droge, ${ }^{1}$ Guenter W. Gross, Mary H. Hightower, and Linda E. Czisny \\ Department of Biological Sciences, North Texas State University, Denton, Texas 76203
}

Laser-deinsulated, printed-circuit electrodes integrated into the floor of culture chambers have been used to monitor the spontaneous activity of mouse spinal monolayer cell cultures. This technique has allowed a multisite analysis of activity over long periods of time in closed chambers. In 63 cultures investigated 3-5 weeks after seeding, 89\% included single- or multiunit bursting. Based on a subset of $\mathbf{4 0}$ cultures in which all electrodes were sequentially scanned, bursting was found on $41 \%$ of the active electrodes (approximately $38 \%$ of all units monitored). A total of $35 \%$ of the electrodes monitoring spontaneous bursting activity revealed rhythmic sequences that were usually coupled among multiple electrodes. Although most of this coupling was in-phase, three out of $\mathbf{4 0}$ cultures exhibited antiphasic bursting. In all cases where coupling was observed, each electrode monitored different burst compositions, demonstrating that the activity was generated by different units. Some rhythmic patterns persisted for over $12 \mathrm{hr}$ and were observed in $400 \mathrm{~mm}^{2}$ monolayer cultures, as well as in much smaller 3 mm$^{2}$ adhesion islands.

The addition of $10 \mathrm{mM} \mathrm{MgCl}_{2}$ consistently blocked both random and patterned (i.e., bursting) spontaneous activity at all recording sites. Strychnine $\left(10^{-6} \mathrm{M}\right)$ typically increased firing frequencies and either disrupted pretest bursting or generated rhythmic activity from random phasic patterns. In certain cases, strychnine also blocked activity on specific electrodes, indicating that glycine is not the only inhibitory transmitter involved.

The spontaneous appearance of rhythmic activity in low-density, monolayer cell cultures established from dissociated and randomly seeded spinal tissue can be explained by one or a combination of two hypotheses: (1) an inherent specificity of some interconnections in developing mammalian cultures and (2) the generation of organized activity by random circuits at certain stages of complexity.

Rhythmic motor activity in vertebrates and in many invertebrates is believed to originate from interneuronal networks (Delcomyn, 1980; Friesen and Stent, 1978; Grillner, 1979; Stein, 1978; Stent, 1983). While such oscillatory networks have been identified in several invertebrates (Getting, 1981; Stent, 1983), the individual components generating vertebrate network rhythms remain unknown. Since vertebrate locomotor and

Received June 27, 1985; revised Jan. 13, 1986; accepted Jan. 13, 1986.

We wish to thank Howard Gartland for his expert tcchnical assistancc. Wc arc grateful for the fabrication of gold multimicroelectrode plates by Siemens Corporation, Munich, in 1977. These plates have been used repeatedly for eight years. We also thank Polytronix Inc., of Richardson, Texas, for their extensive support with the fabrication of the new indium-tin oxide plates. This research was supported by NIH Grant NS 15167 and by an institutional research grant from Texas Woman's University.

Correspondence should be addressed to Dr. Gross at the above address.

' Present address: Department of Biology, Texas Woman's University, Denton, TX 76204.

Copyright $\odot 1986$ Society for Neuroscience $0270-6474 / 86 / 061583-10 \$ 02.00 / 0$ scratching rhythms can persist after the spinal cord is isolated from descending and peripheral influences (Grillner and Wallen, 1985; Stein and Grossman, 1980), local circuits must exist that are capable of pattern generation. Consequently, there has been a trend toward further simplification of vertebrate preparations both surgically and pharmacologically (Cohen and Harris-Warrick, 1984; Droge and Leonard, 1983; Grillner and Wallen, 1985; Grillner et al., 1983; McClellan and Farel, 1985; Roberts et al., 1981; Stein and Grossman, 1980). Despite considerable progress, these preparations still retain an enormous complexity that limits the identification of cellular components involved in rhythmic pattern generation. Therefore, it is desirable to explore even simpler model systems capable of generating patterned activity. In this paper we demonstrate that monolayer spinal cultures, which represent a substantial simplification of CNS tissue, contain the elements necessary for rhythmogenesis and may be used for investigating the fundamentals of pattern generation.

The existing knowledge of circuit development and activity in culture is limited. Despite elegant experiments (Crain, 1974, 1976; Crain and Bornstein, 1972; Fischbach and Nelson, 1977; Harvey, 1984; Nelson and Lieberman, 1981), conventional recording techniques have not allowed a simultaneous monitoring of the activity occurring at numerous sites within a neuronal ensemble. While repetitive, synchronous spike activity has been reported in dissociated spinal cultures (Crain, 1976; Harvey, 1984; Nelson and Lieberman, 1981; Ransom et al., 1977b), only short sequences have been documented. The possibility that rhythmic activity in mammalian spinal cultures is a ubiquitous phenomenon that persists for days and perhaps weeks has not yet been systematically studied. Moreover, the hypothesis that this activity primarily results from network properties rather than endogenous bursters has not been addressed.

The present report demonstrates prolonged, coordinated sequences of rhythm in mammalian, monolayer cultures. The data were obtained with a new procedure that allows simultaneous, long-term, multiunit recording with photoetched multielectrode plates (MMEPs) (Gross and Lucas, 1982; Gross et al., 1985). This work is primarily based on investigations of activity in $400 \mathrm{~mm}^{2}$ monolayer cultures. However, sample data from rhythmic networks in significantly smaller cultures $\left(3 \mathrm{~mm}^{2}\right)$ have also been included. This report documents the occurrence of patterned bursting, as well as rhythms, in monolayer cultures of fetal mouse spinal tissue and argues that this complex activity is primarily a network phenomenon.

\section{Materials and Methods}

\section{Cell culture}

Whole spinal cords were excised from 13-14-d-old mouse embryos and prepared according to the method of Ransom et al. (1977a), with the addition of an enzymatic dissociation step ( $30 \mathrm{~min}$ in $0.25 \%$ trypsin) before trituration. Volumes of $1.0 \mathrm{ml}$ containing approximately $1 \times$ $10^{6}$ cells (neurons and glia) were added to multimicroelectrode plates 


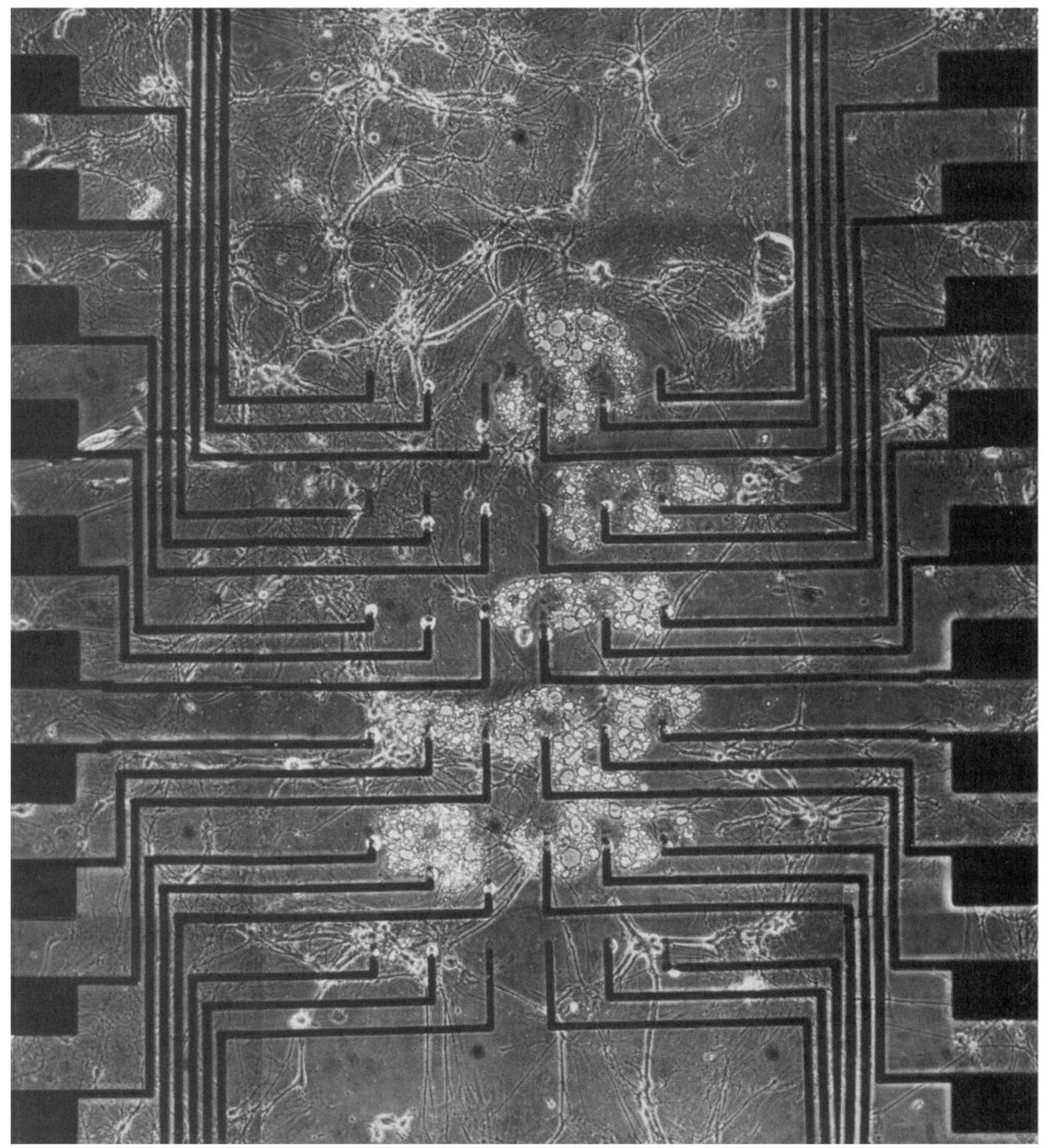

Figure 1. Phase-contrast micrograph of a multielectrode recording matrix consisting of $10-\mu \mathrm{m}$-wide gold conductors arranged in six rows and six columns. Craters produced by lasing through a polysiloxane insulating resin can be seen at the tips of the conductors and represent the sites for extracellular recording of action potentials. Dissociated, embryonic, mouse spinal tissue, seeded onto the insulated surface 3 weeks earlier, has developed a monolayer network. Some insulation deterioration can be seen, especially along rows 4 and 5 . In this particular network, 17 of 28 functional electrodes monitored spontaneous activity.

(MMEPs) and confined to $400 \mathrm{~mm}^{2}$ areas with silicone gaskets. The initial medium consisted of Modified Eagle's Medium (MEM) (Gibco) supplemented with $10 \%$ fetal bovine serum and $10 \%$ horse serum. After $4 \mathrm{~d}$ in culture, the fetal bovine serum component was discontinued. Fluorodeoxyuridine and uridine were added to the plates at $5 \mathrm{~d}$ postseeding in concentrations recommended by Ransom et al. (1977a), in order to reduce the total number of glia and restrict subsequent glial proliferation. Complete medium changes were performed 1 and $4 \mathrm{~d}$ postseeding; thereafter, $50 \%$ medium changes were carried out biweekly. The concentrations of compounds in the medium known to directly affect electrical activity were $\mathrm{K}^{+}, 4.8 \mathrm{~mm} ; \mathrm{Ca}^{2+}, 4.5 \mathrm{~mm}$; glycine, 8.0 $\mu \mathrm{M}$; aspartate, $0.3 \mu \mathrm{M}$; and glutamate, $1.5 \mu \mathrm{M}$. No antibiotic or antimycotic compounds were used during the seeding and development of the cultures or during recording. 

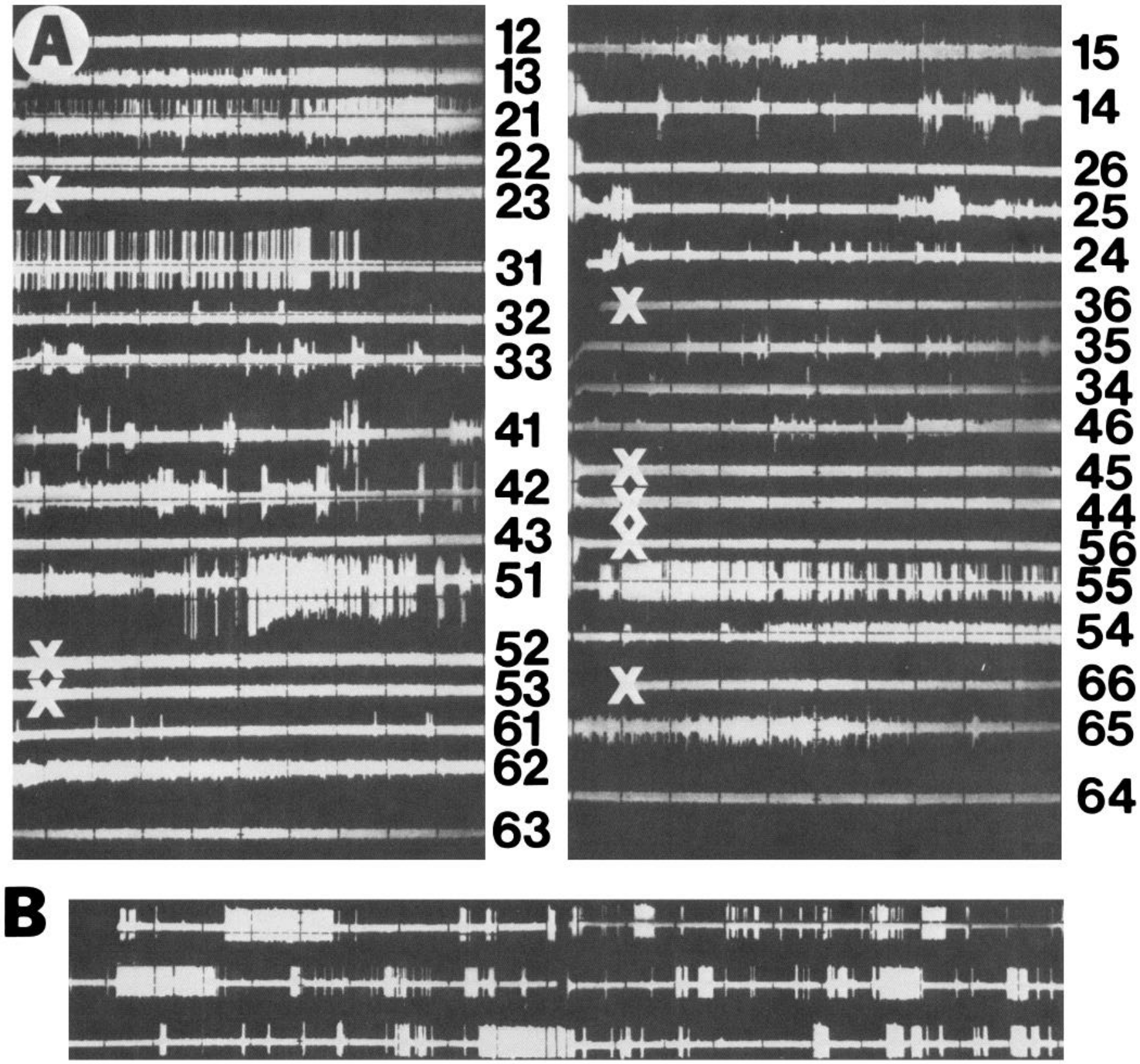

Figure 2. A, Activity patterns from one culture sampled $34 \mathrm{~d}$ after seeding. The data show sequential oscilloscope sweeps ( 2 sec/div) stored at 40 sec intervals and do not represent simultaneous activity. In this example, bursting is evident on at least 16 of 24 functional electrodes or on 16 of 20 electrodes carrying discernible activity. The numbers to the right of each panel represent the row-column identifiers of each electrode. $\mathrm{E} 63$ (sixth row, third column) and E 64 were left insulated to allow a monitoring of shunt-impedance stability. The symbol $\times$ denotes electrodes inactive because of conductor discontinuities. $B$, Samples of complex single-unit bursting recorded from E 31 over a 5 min period (sweep, 5.0 sec).

\section{Multimicroelectrode plates}

The characteristics of gold MMEPs and their insulation and laser deinsulation have been previously described (Gross, 1979; Gross and Lucas, 1982). Figure 1 shows a 3-week-old culture grown on a gold MMEP. The thin-film conductors in the center are $10 \mu \mathrm{m}$ wide and are spaced $100 \mu \mathrm{m}$ between columns and $200 \mu \mathrm{m}$ between rows. The laser deinsulations through the polysiloxane insulation material can be seen as halos at the ends of the conductors. A medium-density culture (about 300 neurons $/ \mathrm{mm}^{2}$ ) has formed a monolayer network on the surface of the insulation layer. Insulation deterioration due to an insufficient cleaning of the glass surface can be seen, especially along rows 4 and 5 (Fig. 1). It should be noted that optical information is lost at the gold conductors, and any attempt to increase electrode density would be associated with a serious and eventually unacceptable loss of morphological data. This problem is eliminated by the use of transparent indium-tin oxide (ITO) as a conductor material (Gross et al., 1985).

\section{Neuronal adhesion to insulation layer}

The polysiloxsane insulation material used routinely for MMEP fabrication is hydrophobic. It does not accept surface modification with collagen or polylysine until the surface is exposed briefly to a propane flame (Lucas et al., 1986). Specific adhesion patterns as small as 1.0 $\mathrm{mm}$ in diameter can be produced by flaming through a mask. Thereafter, a culture can be successfully grown on a small adhesion island if larger, medium-conditioning areas are provided on the same surface. The hydrophobic region separating these areas prevents neurite migration and maintains electrophysiological isolation of the small culture.

\section{Classification of activity}

Monolayer cell cultures produce a great variety of spontaneous activity, including tonic and phasic firing. It is convenient to subdivide the latter into random phasic and several bursting categories. Random phasic activity consists of single or multiple units firing with a temporal irreg- 


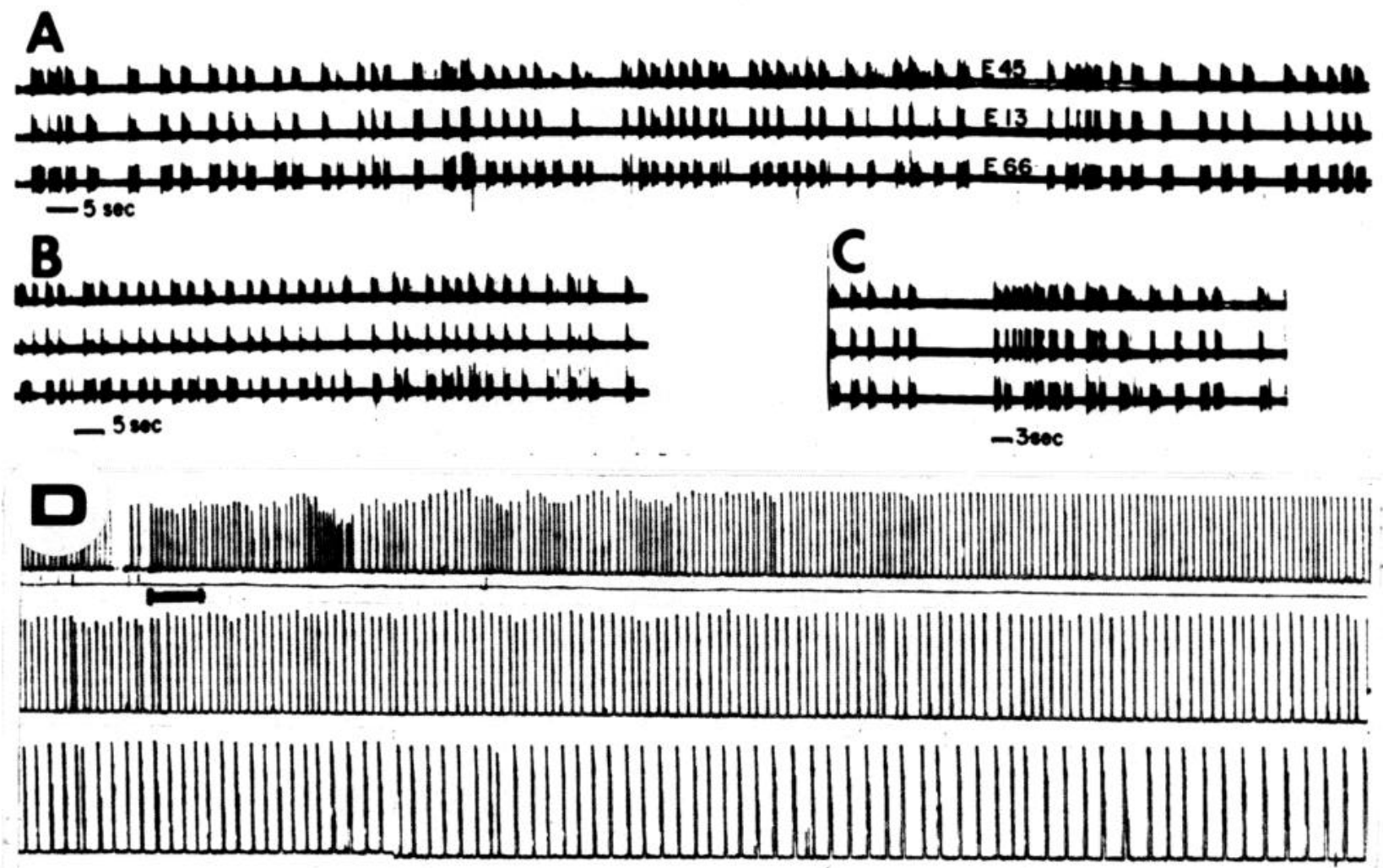

Figure 3. $A-C$, Simultaneous recordings of patterned activity on three electrodes (identified in $A$ ). E 13 and $\mathrm{E} 66$ represent electrodes at opposite ends of the recording matrix separated by $1 \mathrm{~mm}$; E 45 is located near the center. Average values for cycle periods of activity on E 45 for panels $A-C$ are $3.3 \pm 0.8,3.0 \pm 0.5$, and $2.6 \pm 0.8 \mathrm{sec}$, respectively. The CVs are 25,17 , and $30 \%$, respectively. According to criteria described in Materials and Methods, the activity in $C$ is not considered rhythmic. $D$, Continuous chart records of integrated activity over an 82 min period, showing a gradual change in rhythmic bursting from 7.4 to 2.4 cycles $/ \mathrm{min}$. Time bar, $1.0 \mathrm{~min}$.

ularity that prevents the recognition of patterns. A burst, however, is a multispike event of sufficiently high frequency to be clearly separated from previous and subsequent events. In our cultures, the repetition rate of discrete bursts rarely exceeded $50 \mathrm{~Hz}$. Therefore, most of the high-frequency bursting activity was similar to the records shown in Figure $5 G$ of Results. This has led us to a useful criterion for bursting: Interburst intervals should exceed interspike intervals by at least a factor of 3. In our system, smaller interburst intervals make the identification of bursting extremely difficult.

Bursting activity can be regular or irregular in timing. Absolute regularity is probably never generated in neuronal systems; however, highly regular activity occurs and can be quantitatively described by an average cycle period and SD. Because highly regular activity is often disrupted by sequences of irregular bursting, it is necessary either to increase the $\mathrm{SD}$ to encompass all disruptive cycles or to focus on the regular bursting activity. We consider the latter approach more useful in that it can extract rhythmic sequences over long time periods. This is valid as long as the disruptive patterns represent a minority of the total number of cycle periods. For a quantitative determination of the occurrence of rhythm, we have used the following arbitrary, yet practical, procedure: (1) determine the average cycle period from a selected sequence of at least 10 bursts; (2) eliminate $25 \%$ of the most extreme deviations in cycle period; (3) determine a new average and SD; and (4) accept this sequence as rhythmic if the coefficient of variation $(\mathrm{CV}$, i.e., the SD as a percentage of the average) does not exceed $25 \%$ of the average. Since the time period selected for analysis will influence both the average cycle period and $\mathrm{CV}$, sequences having many more than 10 bursts (up to several hundred) were preferentially chosen.

\section{Results}

Typical spontaneous bursting patterns from a culture sampled $34 \mathrm{~d}$ after seeding are shown in Figure 2. The data in Figure $2 \mathrm{~A}$ show sequential oscilloscope sweeps stored separately at approximately $40 \mathrm{sec}$ intervals. Because of cuts in the thin-film electrode pattern, only 24 of the 36 electrodes were functional. Of these, $20(83 \%)$ carried discernible activity. Bursting is evident on at least 16 of the 20 active electrodes (80\%). Although not all cultures displayed this high percentage of bursting activity, it was common among cultures older than 4 weeks. Based on the criteria described in Materials and Methods, none of the activity in this culture could be classified as rhythmic during the time sampled. Even when the single-unit activity of electrode 31 was monitored for a $5.0 \mathrm{~min}$ period (Fig. $2 B$ ), the pattern, despite considerable complexity, was not rhythmic.

While a variety of tonic and phasic activity was usually observed, closer examination of 63 MMEPs with a $400 \mathrm{~mm}^{2}$ culture area revealed spontaneous bursting on at least one electrode in $89 \%$ of the cultures. Based on a subset of 40 MMEPs, in which all electrodes were sequentially scanned, we found bursting activity on $41 \%$ of the active electrodes. As some of these electrodes recorded activity from more than one unit, a further normalization revealed that $38 \%$ of all active units monitored showed bursting activity. It should be noted that this simple analysis underestimates the actual number of bursting units due to the intermittent nature of some bursting activity and the relatively short scanning periods.

Whenever patterned bursting activity was observed, it was monitored for at least $15 \mathrm{~min}$. From those data, regular bursting sequences were analyzed according to our criteria for rhythmicity. This analysis revealed that $35 \%$ of all bursting electrodes ( $14 \%$ of all active electrodes) displayed rhythmic patterns. The observed rhythms ranged from brief episodes to sustained sequences having hundreds of cycles (Fig. 3). For three examples of patterned activity shown (Fig. $3, A-C$ ), the average cycle periods, SDs, and concomitant CVs were $3.3 \pm 8.0 \mathrm{sec}, 25 \%$; 
$3.0 \pm 0.5 \mathrm{sec}, 17 \%$; and $2.6 \pm 0.8 \mathrm{sec}, 30 \%$, respectively. Accordingly, Figure $3, A$ and $B$, displays rhythmic activity, whereas Figure $3 C$ represents patterned, nonrhythmic activity. The fourth example (Fig. 3D) shows a continual decrease in firing frequency from 7.4 to 2.4 cycles/min. Disregarding the first several minutes shown, the activity is rhythmic regardless of the interval chosen. Several cultures exhibited apparently persistent, spontaneous rhythms for days. The overall range of cycle periods across all identified rhythms was $4 \mathrm{msec}$ to $3 \mathrm{~min}$. The two groups of cycle periods most often observed included (1) cycles of $50 \mathrm{msec}$ or less $(28 \%)$, and (2) cycles ranging from 1 to $10 \mathrm{sec}(57 \%)$. Nearly three-fourths of the cycles within the second group were of $1-3 \mathrm{sec}$ duration.

Rhythmic bursting usually included in-phase coupling among multiple recording sites. Such activity was observed on up to 18 electrodes dispersed over a $0.5 \times 1.0 \mathrm{~mm}$ area. Most electrodes monitored several active units responding with different burst compositions in synchrony. There was no tendency for coupled activity to be restricted to closely positioned electrodes. In Figure 3, coupled recordings are shown from electrodes in rows 4,1 , and 6 and columns 5, 3, and 6, respectively. Electrodes 13 and 66 are located at nearly opposite ends of the recording matrix, representing a separation of about $1 \mathrm{~mm}$.

Despite a striking synchrony of bursting activity among various regions of the cultures (e.g., Fig. 3), antiphasic (alternating) activity was found in three out of 40 cultures (Fig. 4). Although rare, it demonstrates that these cultures can attain a level of circuit complexity sufficient for the generation of reciprocal patterns.

Figure 5 shows the great variety of burst compositions and frequencies generated by monolayer cultures. The majority of all rhythmic sequences $(81 \%)$ had bursts consisting of fewer than four different units (usually axons). Typically, the units producing such activity collectively fired fewer than 10 spikes per burst. However, cycle periods of several seconds or longer occasionally had bursts with more than 100 spikes. Rhythm produced by one or two units firing numerous times per burst was frequently observed (e.g., Fig. 5). This result was not surprising, considering the recording arrangement and low-density morphology of the monolayer cultures. Often only a single or a few axons crossed any given recording crater, which could not detect activity from axons $10 \mu \mathrm{m}$ beyond its edge. As shown in Figure $5 C$, the action potentials consistently had smooth, monophasic repolarizations, and thus the appearance of $\mathrm{Na}^{+}$spikes. The bursting shown in Figure $5, F-H$, represents one of the highest bursting frequencies observed $(25-30 \mathrm{~Hz})$. This particular activity was stable over a long period of time and was observed at two monitoring intervals separated by $18 \mathrm{hr}$.

In all of 12 experiments, the addition of $10 \mathrm{mM} \mathrm{MgCl}_{2}$ to the medium resulted in an abrupt cessation of all spontaneous activity (Fig. 6). This effect was reversible by replacing the $\mathrm{MgCl}_{2}$ solution with normal medium, although burst composition and pattern often changed. The introduction of $10^{-6} \mathrm{M}$ strychnine typically increased the firing rate of all types of activity and either disrupted pretest bursting or generated rhythmic activity from random phasic patterns. However, strychnine was also observed to have a differential effect at separate recording sites. Figure 7 shows that, after strychnine administration, the activity seen at two recording sites increased, while that of a third site (recorded simultaneously) was inhibited.

The simplification represented by monolayer cultures is still not sufficient for quantitative analyses as long as the cultures greatly exceed the size of the recording matrix. Under those conditions, the activity being monitored may originate from neurons located nearly $1.0 \mathrm{~cm}$ from the recording craters. This makes the visual identification of network components, as well as the network mapping after fixation, very difficult. It is therefore necessary to grow networks in much smaller culture areas
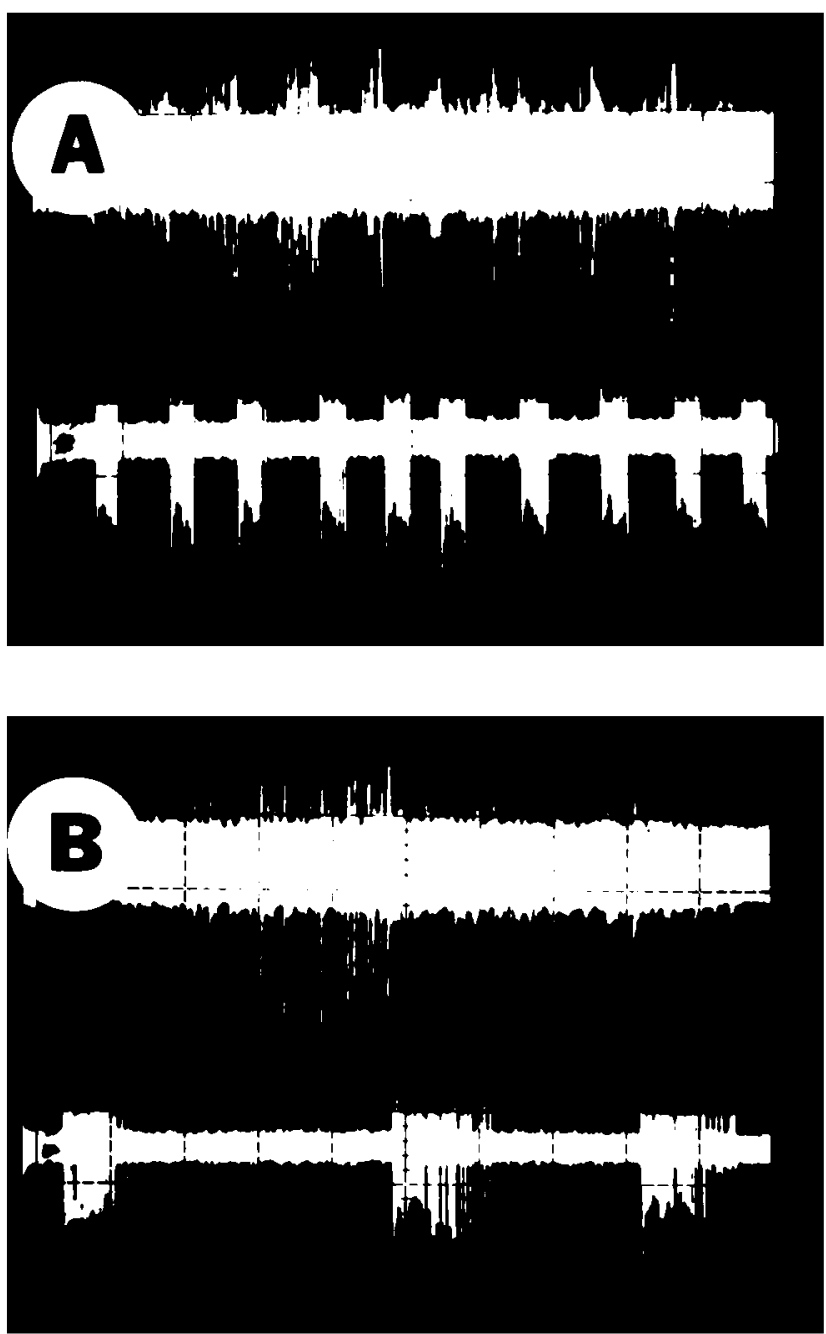

Figure 4. Antiphasic coupling between bursting activity on two electrodes separated by $675 \mu \mathrm{m}$. Sweep speeds for $A$ and $B$ are 2.0 and 0.5 $\mathrm{sec}$, respectively. This activity was obtained from a 2 -mm-diameter adhesion island containing about 400 neurons. An example of such a small culture is shown in Figure 8.

for a further simplification of electrophysiological and morphological analyses.

Figure 8 shows a 1.8 -mm-diameter, Bodian-Nissl-stained culture centered on the 36 electrode recording matrix of a goldconductor MMEP. The regular conductor array was deteriorated as a result of repeated cycles of insulation and deinsulation over an 8 year period. However, 21 electrodes were still functional, 16 displaying spontaneous, coupled bursting activity. This culture contained approximately 400 neurons, tentatively identified by their Nissl staining and morphological characteristics (Hightower and Gross, 1985). Integrated activity from this culture is presented in Figure 9. Although the spontaneous activity in normal medium revealed an irregular bursting pattern, the addition of strychnine (Fig. 9B) triggered a long period of rhythmic bursting. It should be noted that this culture was electrophysiologically isolated from other culture areas on the same electrode plate. The latter areas (not shown in Fig. 8) appear to be required for medium conditioning and are typically arranged as two $100 \mathrm{~mm}^{2}$ regions to either side of the recording matrix. The separation between the recording and conditioning areas is $1.0 \mathrm{~cm}$, and no neurites have been observed to cross the hydrophobic surface between the adhesion areas. 

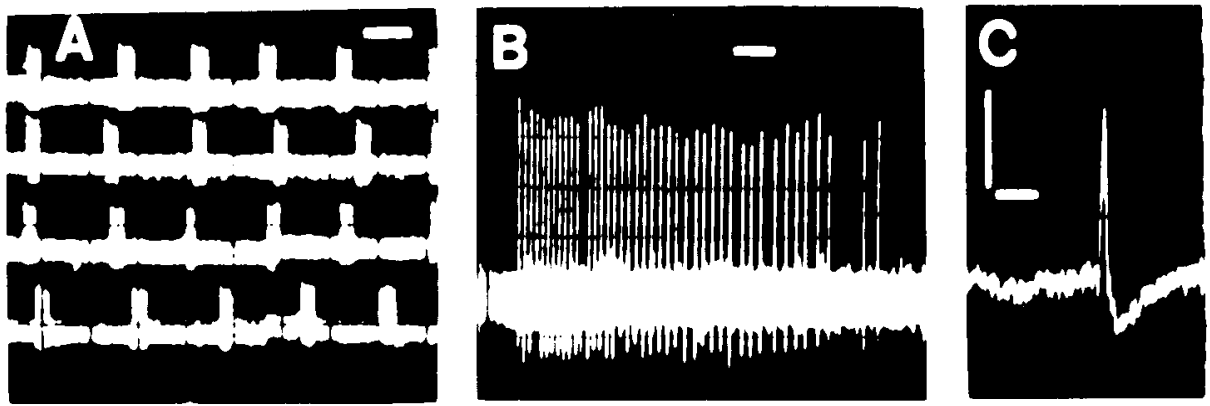

Figure 5. Examples of variety in burst composition and frequency. $A$, Continuous records of a single-unit rhythmic sequence; sweep, $5.0 \mathrm{sec} . B$, Example of an individual burst in $A$; sweep, $0.2 \mathrm{sec}$. $C$, Example of a single spike from the bursts shown in $A$ and $B$; sweep, $2.0 \mathrm{msec}$ (vertical calibration, $100 \mu \mathrm{V}$ ). $D$ and $E$, Examples of frequently observed complex, multiunit bursts; sweep, $50 \mathrm{msec}$ for each. $F$, Very rapid rhythmic sequence (25 Hz); sweep, 0.2 sec. $G$ and $H$, Bursts taken from the sequence in $F$ to show burst composition; sweep, 20 and 2 $\mathrm{msec}$, respectively. Bandwidth for all figures, $100 \mathrm{~Hz}-10 \mathrm{kIIz}$.
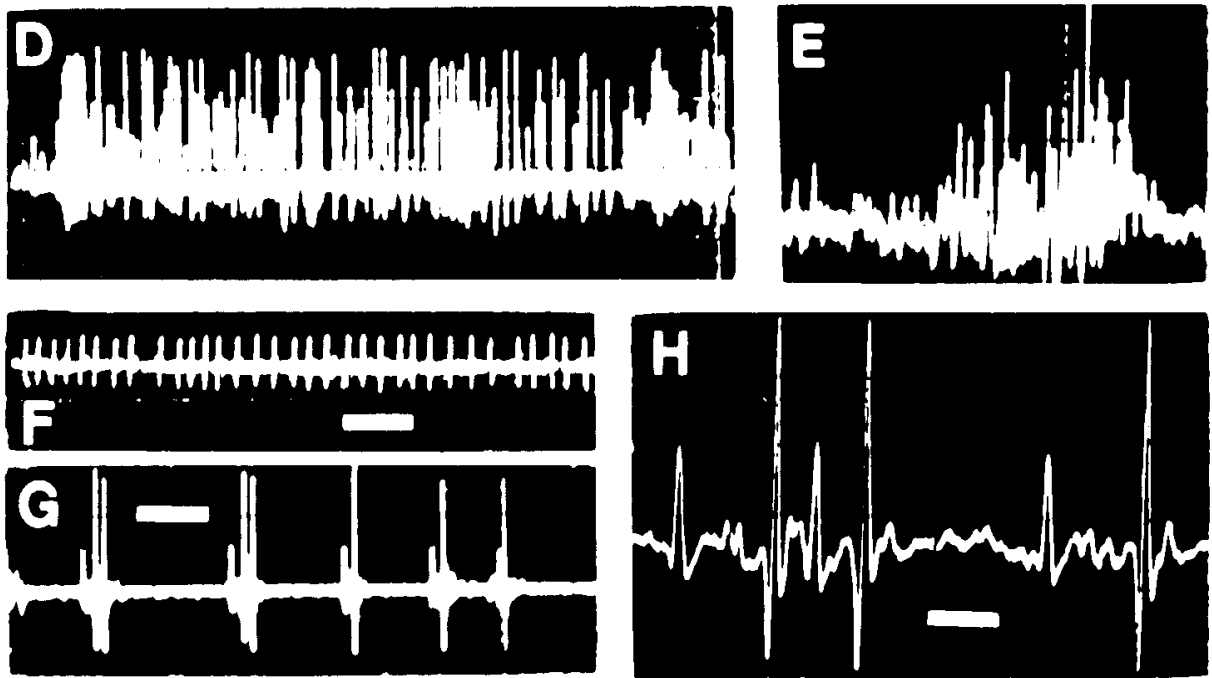

\section{Discussion}

According to MacDonald and Barker (1981), the spontaneous activity in cultured spinal neurons usually consists of random action potentials. In contrast, we found bursting sequences to be a common occurrence ( 182 of 443 active electrodes, or $41 \%$ ). Of the electrodes monitoring bursting activity, $35 \%$ revealed rhythmic sequences based on the relatively strict criteria described in Materials and Methods. It is likely that our observation of rhythms was primarily a result of the recording techniques employed. Compared to conventional techniques, recordings with printed-circuit, multielectrode patterns provide a more global view of culture activity. Also, since the cultures grow on the electrode matrix, recording is possible with a minimal disturbance of the neuronal circuitry. A review of the literature, however, confirms that alternating quiescence and paroxysmal activity has been observed from single spinal neurons in cultures ( $c f$. Crain, 1976; Nelson and Lieberman, 1981). Synchronous, repetitive spike activity has also been documented for two connected neurons (Nelson et al., 1981). However, these activities were not shown to persist for long periods.

It is clear from Figures 3, 5, and 9 that repetitive bursting can occur in monolayer cultures with surprisingly consistent cycle periods. In many cases, however, sequences were observed that exhibited varying degrees of regularity and comparatively slow rhythms (Fig. 6). It is interesting that the cycle periods of rhythms generated by intact spinal cords are often considerably increased when phasic afferent feedback is removed pharmacologically (Droge and Leonard, 1983). It is also interesting that small, monolayer networks in culture (300-500 neurons) can generate alternating activity, which is a basic feature of rhythms observed in intact spinal cords.

Chemical synapses are considered essential for pattern generation in various systems. It is widely accepted that vertebrate locomotor rhythms arise from local interneuronal circuits within the spinal cord (Delcomyn, 1980; Grillner, 1975; Grillner and Wallen, 1985; Stein, 1978). A requirement for chemical synaptic transmission in rhythmogenesis has also been dem-

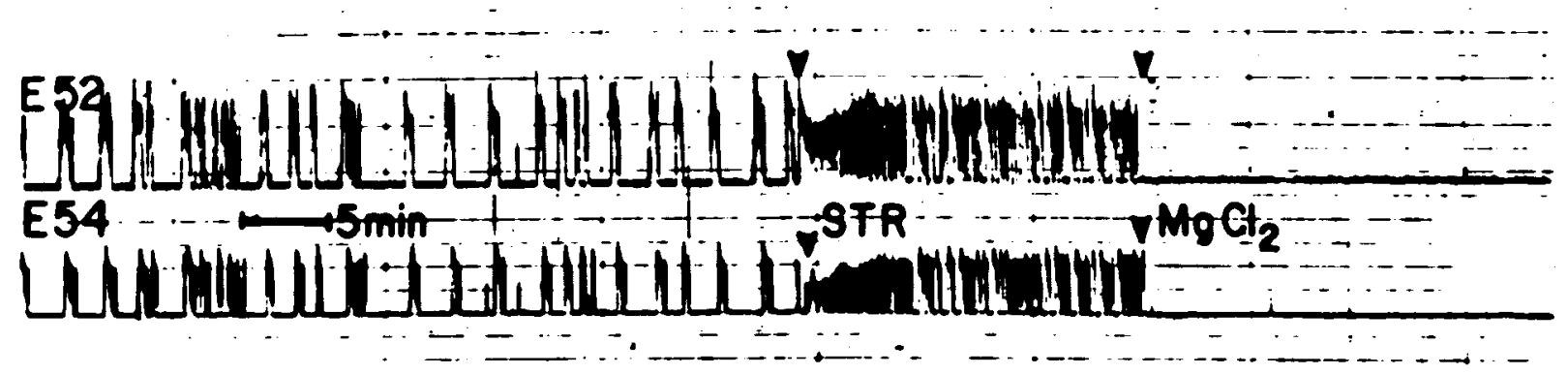

Figure 6. Integrated, simultaneous records from two electrodes separated by $200 \mu \mathrm{m}$, showing the effects of strychnine and $\mathrm{MgCl}_{2}$ on spontancous, coordinated activity. First arrow, Bath application of $10^{-6} \mathrm{M}$ strychnine; second arrow, bath application of $10 \mathrm{mM} \mathrm{MgCl}_{2}$. 


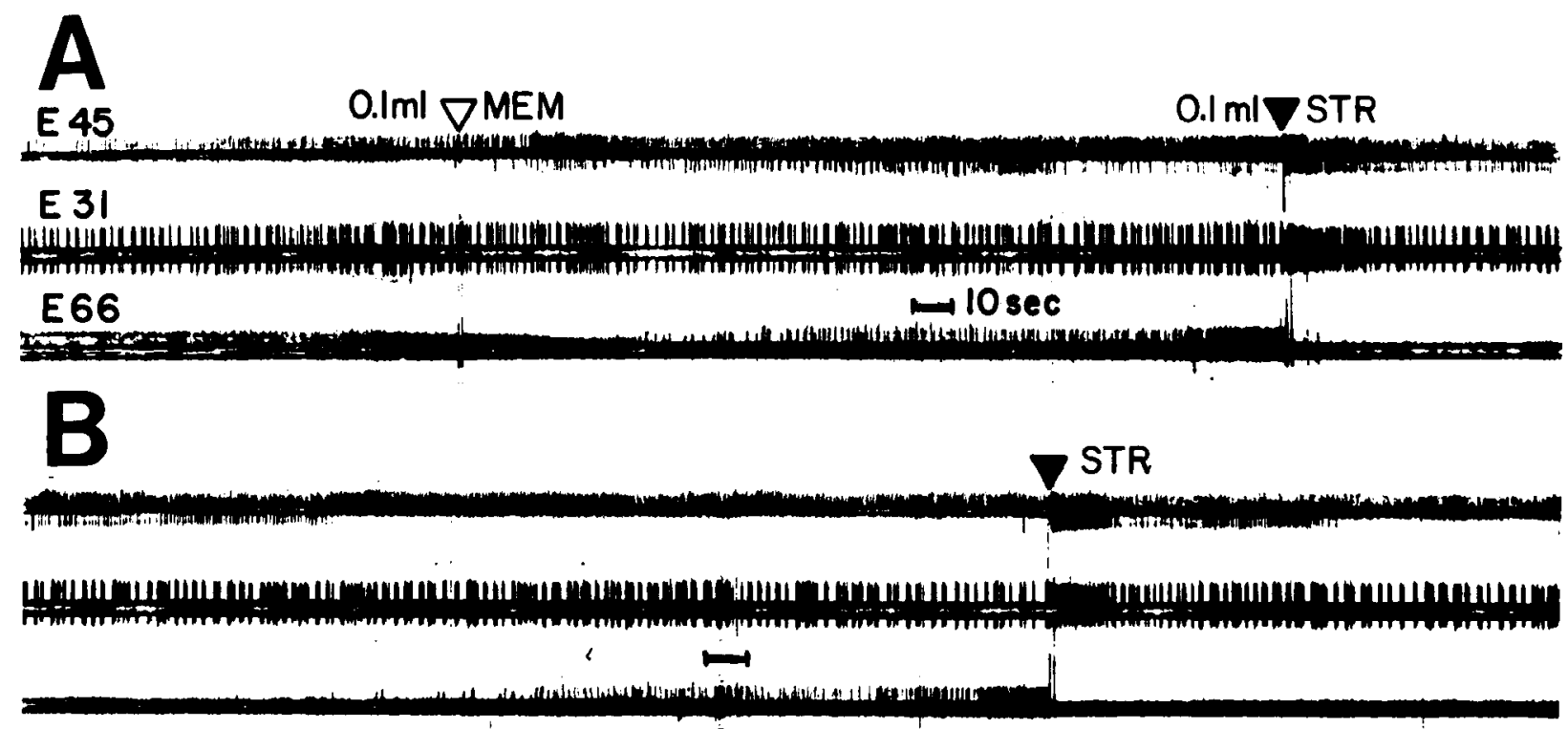

Figure 7. Differential effect of strychnine on multiple simultaneous recordings. $A$, Spontaneous activity at three different recording sites. Open arrow, Control application of fresh medium (MEM); filled arrow, the addition of $10^{-6} \mathrm{M}$ strychnine. $B$, Another application of strychnine 3 min after the previous test $(A)$, showing a reproducible, differential effect.

onstrated for hippocampal slices (Oliver et al., 1978). The authors cited above showed that spontaneous, sustained, rhythmic, interictal spiking required intact synaptic transmission in addition to increased extracellular potassium and a convulsant drug. Considerable evidence also indicates that spontaneous activity in cultures grown from dissociated fetal mouse spinal tissue primarily involves chemical synaptic transmission (Harvey, 1984; Nelson et al., 1977, 1981; Ransom et al., 1977b). Both excitatory and inhibitory postsynaptic potentials have becn reported in culture (Nelson et al., 1981). After testing the effects of depolarizing currents on EPSP amplitude, Ransom et al. (1977b) concluded that the EPSPs recorded between cultured spinal neurons were chemically mediated. Finally, while searching for clectrical coupling in over 200 pairs of spinal cord ncurons in culture, the same authors found only one case of such coupling. The single pair that was electrically coupled (bidirectionally) also had excitatory chemical synapses.

Additional support for the involvement of chemical synaptic transmission in activity recorded from cultures is based on the manipulation of divalent ions, especially $\mathrm{Mg}^{2+}$ (Crain, 1976). Ransom et al. (1977b) demonstrated that a $10 \mathrm{~mm}$ extracellular $\mathrm{MgCl}_{2}$ concentration blocked all PSPs in mouse spinal cultures. While this effect suggests the involvement of synaptic transmission, such data alone are inconclusive. In addition to interfering with transmitter release, increased $\mathrm{Mg}^{2+}$ (and possibly other divalent ions) may also raise spike thresholds of neurons (Ransom et al., 1977b). If so, a rise in spike threshold could suppress spontaneous activity resulting from electrical as well as chemical synapses. This may explain why we have not observed a continuation of any patterned or random spontaneous activity following the administration of $\mathrm{MgCl}_{2}$.

Conclusions regarding the existence of endogenous bursters are difficult. Subthreshold oscillations of membrane potential could not be monitored with our extracellular electrodes. If endogenous bursters are not affected by the $10 \mathrm{mM} \mathrm{MgCl}$, the increased number of recording sites monitored per culture should eventually demonstrate persistent activity. Since this has not occurred in 12 experiments, two other alternatives should be considered: (1) endogenous bursters do not exist in these cultures, or (2) $\mathrm{MgCl}_{2}$ raises the threshold sufficiently to block endogenous spike generation. It is not known if the rise in threshold affects only $\mathrm{Na}^{+}$spikes or if it also applies to $\mathrm{Ca}^{2+}$ spikes, as was described for the classical model of endogenous bursters (Kristan, 1980). These endogenous bursters involve slow, $\mathrm{Ca}^{2+}$-dependent conductance changes, and they show bursting even when all synaptic activity is blocked. In view of the evidence that all spontaneous intracellular activity in dissociated mouse spinal cultures is TTX-sensitive and therefore $\mathrm{Na}^{+}$-dependent (Harvey, 1984; Nelson and Lieberman, 1981; Ransom et al., 1977b), it appears that the influence of such endogenous bursters may be limited. It remains possible, however, that endogenous oscillators provide perturbations that trigger activity, the rhythmic character of which is primarily determined by network properties.

Our finding that low doses of strychnine greatly enhanced spontaneous activity has also been reported in other systems (Harvey, 1984). Since strychnine specifically blocks glycine synapses, this enhancement of activity is considered a synaptic event (Cohen and Harris-Warrick, 1984; Grillner and Wallen, 1980; Homma and Rovainen, 1978). The increases in firing frequency can be explained as disinhibition of excitatory synapses via glycine transmission (Cohen and Harris-Warrick, 1984; Grillner and Wallen, 1980). Such evidence supports the hypothesis that networks generating rhythm in certain vertebrates involve recurrent inhibition (Kling and Szekely, 1968; Szekely, 1965). Cyclic inhibitory circuits have also been reported for locomotor rhythms in an invertebrate (Friesen and Stent, 1977). However, the differential effect of strychnine observed so far on a few electrodes (Fig. 7) indicates that glycine transmission is not the only inhibitory transmitter involved. This cessation of activity may represent a disinhibition via glycine that increases activity in other types of inhibitory connections. Since these observations are rare (two of 12 cultures), the contention of other investigators that glycine inhibition predominates in these preparations appears to be valid (Jackson et al., 1982; Nelson et al., 1977).

Certain evidence suggests that some of the interconnections made in culture may be specific. The development of "organotypic" activity in some dissociated cell cultures was thought to represent specificity in synaptogenesis (Crain, 1976; Crain and 


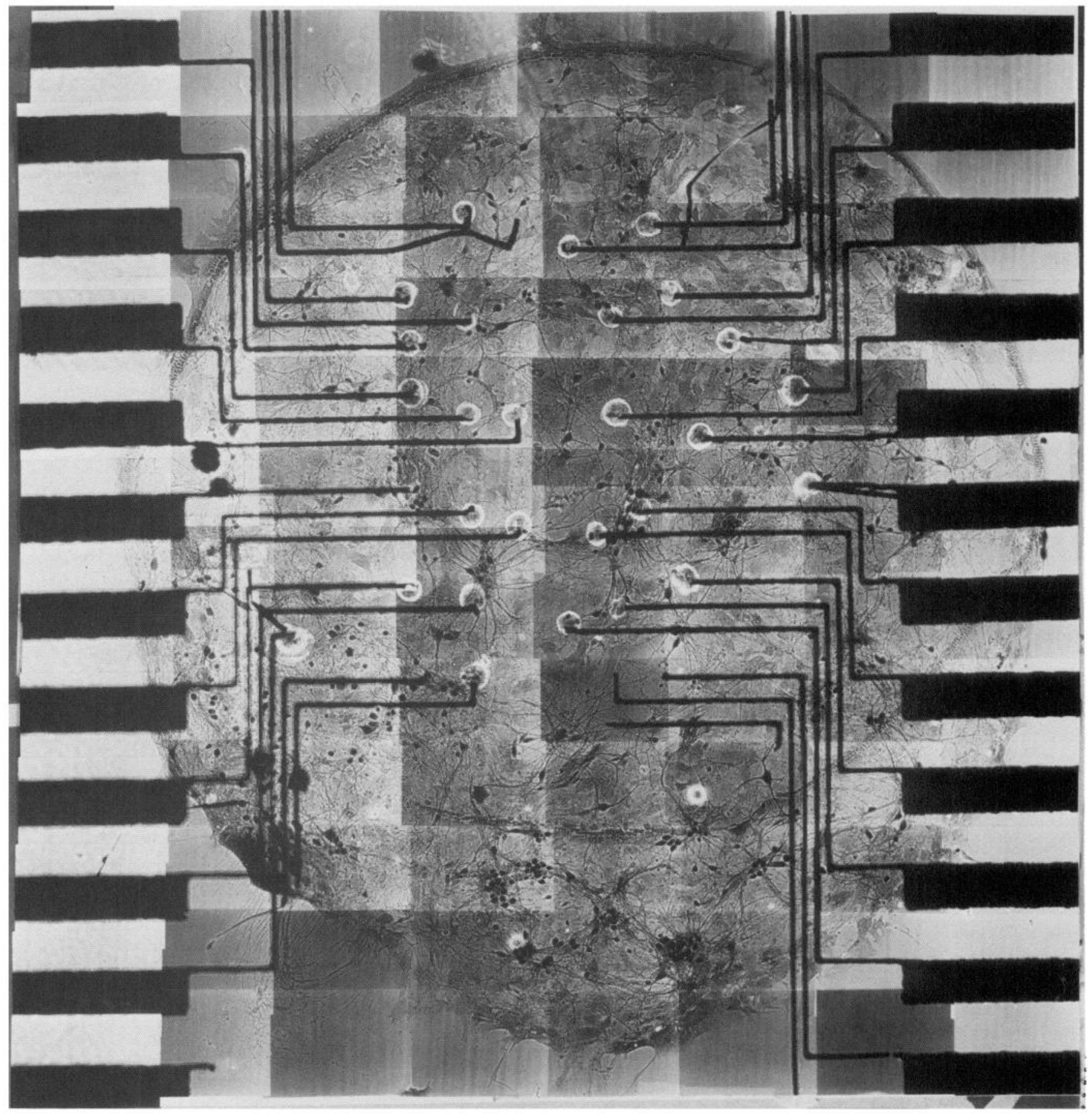

Figure 8 . Monolayer adhesion island (1.8 mm diameter) centered over recording matrix. The Bodian-Nissl-stained culture contained approximately 400 neurons. Spontaneous bursting activity was identified on 16 of 21 electrodes. The bursting activity on all electrodes was coupled. This MMEP was fabricated in 1977 and shows a general deterioration of the $10-\mu \mathrm{m}$-wide gold conductors.

Bornstein, 1972; Fischbach and Dichter, 1974). Fischbach and Dichter found that functional contacts are sometimes made among distant neurons in preference to immediately adjacent cells. Among invertebrates, cultured identified neurons from the abdominal ganglion of Aplysia californica avoid making functional contacts with cells that are not targets in their in situ circuitry (Camardo et al., 1983). However, more recent investigations imply that synaptogenesis in vertebrates is much more complex and may be primarily influenced by competition among terminals, trophic feedback, and modifications due to functional activity ( $c f$. Easter et al., 1985; Edelman, 1983). The question remains, however, whether such a degree of complexity can be present in spinal monolayer cultures, where muscular and glandular target tissues, as well as DRG cells and some target neurons, are absent. Furthermore, without any sensory input or systematic electrical stimulation, "functional activity" cannot be defined.

If the phenomenon of synaptogenesis in these spinal cultures is indeed simpler than that observed in situ, and if specific synaptogenesis via recognition molecules (i.e., strict chemoaffinity) is not a major factor, we are faced with the prospect that random circuits generate the observed organized activity. It is 

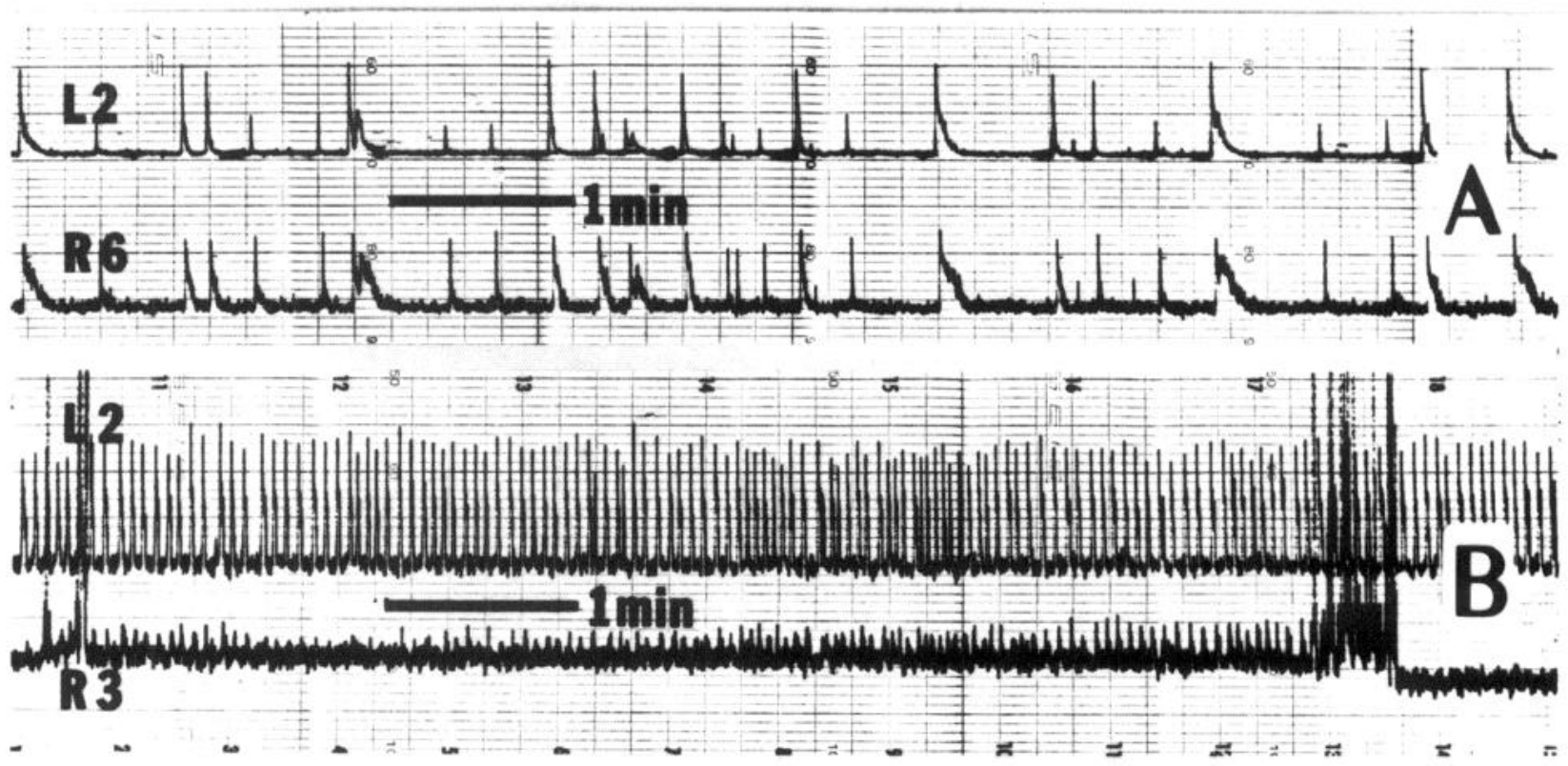

Figure 9. Sample activity obtained from the small monolayer culture shown in Figure 8. A, Spontaneous bursting coordinated between two electrodes (Fig. 8; arrows). B, Rhythmic activity at 17 bursts/min following administration of $10^{-6} \mathrm{M}$ strychnine. Average cycle period ( \pm SD), $3.5 \pm 0.5 \mathrm{sec} ; \mathrm{CV}, 14 \%$.

conceivable that multiple functional circuits could be activated within a larger, randomly formed morphological network. In fact, it has been suggested that "computational tasks" could spontaneously arise in a large number of interacting "simple neurons" (Hopfield, 1982). However, such theoretical considerations have not yet been experimentally confirmed, and it would be premature to use our data as evidence for concepts of self-organization. Certainly, it must still be considered possible that at least the most basic of networks (or functional circuits) are initially influenced by specific membrane-recognition phenomena. Subsequent modulation of synaptic connectivity by sensory feedback, however, is absent and cannot contribute to network stabilization in our cultures. This may explain, in part, why, despite observations of persistent rhythms, much of the activity displays a great variety of phasic bursting and appears to drift among various patterns. It would be very interesting to determine in future experiments whether systematic electrical stimulation could serve to stabilize specific activity patterns.

\section{References}

Camardo, J., E. Proshansky, and S. Schacter (1983) Identified Aplysia neurons form specific chemical synapses in culture. J. Neurosci. 3: 2621-2629.

Cohen, A. H., and R. M. Harris-Warrick (1984) Strychnine eliminates alternating motor output during fictive locomotion in the lamprey. Brain Res. 293: 164-167.

Crain, S. M. (1974) Drugs and the Developing Brain, Plenum, New York.

Crain, S. M. (1976) Neurophysiologic Studies in Tissue Culture, Raven, New York.

Crain, S. M., and M. B. Bornstein (1972) Organotypic bioelectric activity in cultural reaggregates of dissociated rodent brain cells. Science 176 : 182-184.

Delcomyn, F. (1980) Neural basis of rhythm behavior in animals. Science 210: 492-498.

Droge, M. H., and R. B. Leonard (1983) Swimming rhythm in decerebrated, paralyzed stingrays: Normal and abnormal coupling. J. Neurophysiol. 50: 178-191.

Easter, S. S., Jr., D. Purves, P. Rakic, and N. C. Spitzer (1985) The changing view of neurospecificity. Science 230: 507-511.

Edelman, G. M. (1983) Cell adhesion molecules. Science 219: 450457.

Fischbach, B. D., and M. A. Dichter (1974) Electrophysiologic and morphologic properties of neurons in dissociated chick spinal cord cell cultures. Dev. Biol. 37: 100-116.

Fischbach, B. D., and P. G. Nelson (1977) Cell culture in neurobiology. In Handbook of Physiology, The Nervous System, Vol. 1, E. R. Kandel, ed., pp. 719-774, American Physiological Society, Bethesda, MD.

Friesen, W. O., and G. S. Stent (1977) Generation of a locomotor rhythm by a neural network with recurrent cyclic inhibition. Biol. Cyber. 28: 27-40.

Friesen, W. O., and G. S. Stent (1978) Neural circuits for generating rhythmic movements. Annu. Rev. Biophys. Bioeng. 7: 37-61.

Getting, P. A. (1981) Mechanisms of pattern generation underlying swimming in Tritonia. I. Neuronal network formed by monosynaptic connections. J. Neurophysiol. 46: 65-79.

Grillner, S. (1975) Locomotion in vertebrates: Central mechanisms and reflex interaction. Physiol. Rev. 55: 247-304.

Grillner, S. (1979) Interaction between central and peripheral mechanisms in the control of locomotion. In Progress in Brain Research, Reflex Control of Posture and Movement, Vol. 50, R. Granit and O. Pompiano, eds., pp. 227-235, Elsevier-North Holland, Amsterdam.

Grillner, S., and P. Wallen (1980) Does the central pattern generation for locomotion in lamprey depend on glycine inhibition? Acta Physiol. Scand. 110: 103-105.

Grillner, S., and P. Wallen (1985) Central pattern generators for locomotion, with special reference to vertebrates. Annu. Rev. Neurosci. 8: 233-261.

Grillner, S., A. McClellan, K. Sizvardt, and P. Wallen (1983) On the spinal generation of locomotion, with particular reference to a simple vertebrate, the lamprey. Birth Defects 19: 347-356.

Gross, G. W. (1979) Simultaneous single unit recording in vitro with a photoetched laser deinsulated gold multimicro-electrode surface. IEEE Trans. Biomed. Eng. 26: 273-279.

Gross, G. W., and J. H. Lucas (1982) Long-term monitoring of spontaneous single unit activity from neuronal monolayer networks cultured on photoetched multielectrode surfaces. J. Electrophysiol. Technol. 9: 55-69.

Gross, G. W., W. Wen, and J. Lin (1985) Transparent indium-tin oxide electrode patterns for extracellular, multisite recording in neuronal cultures. J. Neurosci. Methods 15: 243-252.

Harvey, A. L. (1984) The Pharmacology of Nerve and Muscle in Tissue Culture, Alan R. Liss, New York.

Hightower, M. H., and G. W. Gross (1985) A combined Bodian-Nissl staining procedure for improved morphological network analysis in neuronal cell culture. Stain Technol. 60: 315-320.

Homma, S., and C. M. Rovianen (1978) Conductance increases produced by glycine and gamma-aminobutyric acid in lamprey interneurones. J. Physiol. (Lond.) 279: 231. 
Hopfield, J. J. (1982) Neural networks and physical systems with emergent collective computational abilities. Proc. Natl. Acad. Sci. USA 79: 2554-2558.

Jackson, M. B., H. Lecar, D. E. Brenneman, S. Fitzgerald, and P. G. Nelson (1982) Electrical development in spinal cord cell culture. J. Neurosci. 2: 1052-1061.

Kling, V., and G. Szekely (1968) Simulation of rhythmic nervous activities. I. Function of networks with cyclic inhibition. Kybenetik 5: 89-103.

Kristan, W. B., Jr. (1980) Generation of rhythmic motor patterns. In Information Processing in the Nervous System, H. M. Pinsker and W. D. Willis, eds., pp. 241-263, Raven, New York.

Lucas, J. H., L. E. Czisny, and G. W. Gross (1986) Adhesion of cultured mammalian CNS neurons to flame-modified hydrophobic surfaces. Vitro Cell. \& Dev. Biol. 22: 37-43.

MacDonald, R. L., and J. L. Barker (1981) Neuropharmacology of spinal cord neurons in primary dissociated cell culture. In Excitable Cells in Tissue Culture, P. G. Nelson and M. Lieberman, eds., pp. 81-110, Plenum, New York.

McClellan, A. D., and P. B. Farel (1985) Pharmacological activation of locomotor patterns in larval and adult frog spinal cords. Brain Res. 332: $119-130$.

Nelson, P. G., and M. Lieberman, eds. (1981) Excitable Cells in Tissue Culture, Plenum, New York.

Nelson, P. G., and J. H. Peacock (1973) Electrical activity in dissociated cell cultures from fetal mouse cerebellum. Brain Res. 61:163174.

Nelson, P. G., E. A. Neale, and R. L. MacDonald (1981) Electro- physiological and structural studies of neurons in dissociated cell cultures of the central nervous system. In Excitable Cells in Tissue Culture, P. G. Nelson and M. Lieberman, eds., pp. 39-74, Plenum, New York.

Nelson, P. G., B. R. Ransom, M. Henkart, and P. N. Bullock (1977) Mouse spinal cord in cell culture. IV. Modulation of inhibitory synaptic function. J. Neurophysiol. 40: 1178-1187.

Oliver, A. P., B. J. Hoffer, and R. J. Wyatt (1978) Interaction of potassium and calcium in penicillin-induced interictal spike discharge in the hippocampal slice. Exp. Neurol. 62: 510-520.

Ransom, B. R., E. Neale, M. Henkart, P. N. Bullock, and P. G. Nelson (1977a) Mouse spinal cord in cell culture. I. Morphology and intrinsic neuronal electrophysiologic properties. J. Neurophysiol. 40: 11321150.

Ransom, B. R., C. N. Christian, P. N. Bullock, and P. G. Nelson (1977b) Mouse spinal cord in cell culture. II. Synaptic activity and circuit behavior, J. Neurophysiol. 40: 1151-1162.

Roberts, A., J. A. Kahn, S. R. Soffe, and J. D. W. Clark (1981) Neural control of swimming in a vertebrate. Science 213: 1032-1034.

Stein, P. S. G. (1978) Motor systems, with specific reference to the control of locomotion. Annu. Rev. Neurosci. 1:61-81.

Stein, P. S. G., and M. L. Grossman (1980) Central program for the scratch reflex in the turtle. J. Comp. Physiol. 140: 287-294.

Stent, G. S. (1983) Rhythmic generator circuits in a simple nervous system. Prog. Brain Res. 58: 147-155.

Szekely, G. (1965) Logical network for controlling limb movements in urodella. Acta Physiol. Acad. Sci. Hung. 27: 285-289. 